\title{
Genremischung und Gattungskonflikt Zur episch-dramatischen Doppelphysiognomie von Hofmannsthals »Andreas«-Fragment
}

Wie andere bedeutende Romanprojekte des frühen 20. Jahrhunderts ist Hofmannsthals "Andreas" Fragment geblieben. Die ältere Forschung hat das vielfach als Symptom eines Scheiterns gewertet und noch der Herausgeber der "Kritischen Ausgabe» ist ihr darin gefolgt. ${ }^{1}$ Dagegen wurde in neuerer Zeit geltend gemacht, dass "Andreas" nicht nur im äußerlichen Sinn unabgeschlossen, sondern »seiner inneren Struktur" nach "fragmentarisch $\aleph^{2}$ sei und dass diese »innere Fragmentarik « durch seine "ästhetisch-poetische Eigenart ${ }^{4}$ motiviert werde. Die Dynamiken der Dissoziation und Spaltung, der Entzweiung, Halbierung und Verdoppelung, von denen der Text handelt, kennzeichnen demnach auch seine Entstehung und Form. Diese Neubewertungen weisen insofern über "Andreas" hinaus, als sie den Blick für die Prozesshaftigkeit und den Suchcharakter von Hofmannsthals gesamtem CEuvre schärfen. Es stellt sich heute, nach der Erschließung des Nachlasses, weniger als ein Werk denn als eine Werkstatt dar und zeugt von offenen Schreibprojekten, die über Textgrenzen hinaus fortgesetzt werden und sich in beständiger Auseinandersetzung mit den eigenen Voraussetzungen, Optionen und Zielen befinden. Das heißt jedoch nicht, dass es nicht unterschiedliche Grade des Gelingens gäbe oder dass sich nicht Spannungen und Konflikte ausmachen ließen, die zu Stockungen, Abbrüchen oder Neuansätzen führten. Nachdem die Schlacht um das Fragmentarische als Ausweis

1 Als Gründe werden u.a. Hofmannsthals »Unvermögen als Romancier«, seine »Unsicherheit in der Grundkonzeption" sowie die Entmutigung durch die "niederdrückenden Zeitereignisse[ ] " angeführt (SW XXX Roman, S. 307f.).

2 Angelika Corbineau-Hoffmann, Der Aufbruch ins Offene. Figuren des Fragmentarischen in Prousts "Jean Santeuil« und Hofmannsthals "Andreas". Ein Versuch. In: HF 9, 1987, S. 163-194, hier S. 166.

3 Mathias Mayer, Die Grenzen des Textes. Zur Fragmentarik und Rezeption von Hofmannsthals »Andreas«-Roman. In: Hugo von Hofmannsthal. Neue Wege der Forschung. Hg. von Elsbeth Dangel-Pelloquin. Darmstadt 2007, S. 62-83, hier S. 72.

4 Achim Aurnhammer, Hofmannsthals "Andreas". Das Fragment als Erzählform zwischen Tradition und Moderne. In: HJb 3, 1995, S. 275-296, hier S. 283. 
der Modernität des "Andreas" erfolgreich geschlagen wurde, soll der hofmannthalsche Text auf einen Konflikt hin durchsichtig gemacht werden, der aus der Orientierung an verschiedenen literarischen Gattungstraditionen resultiert und sich in einer Physiognomie ausprägt, die gleichermaßen von epischen wie dramatischen Zügen geprägt ist. Da sich diese Doppelphysiognomie nur an wie immer vorläufig durchgeformten Sequenzen aufzeigen lässt, konzentrieren sich die Ausführungen auf den zwischen September 1912 und August 1913 entstandenen Hauptentwurf zum "Andreas" sowie auf zeitlich benachbarte Aufzeichnungen.

Für das Erzählvorhaben, für das der Name »Andreas«-Roman üblich geworden ist, hat Hofmannsthal nicht nur unterschiedliche Titel, sondern auch unterschiedliche Gattungsbezeichnungen erwogen. Der Begriff "Roman" spielt dabei zunächst keine Rolle. Dieser wird erst nach Abbruch der Arbeit an dem zusammenhängenden Text des Hauptentwurfs eingeführt. Der erste Beleg findet sich - sieht man von einem Brief Hofmannsthals an seinen Vater ab, in dem die Referenz des Begriffs offenbleibt $-^{5}$ in einer Aufzeichnung zum Finazzerhof vom Herbst 1913. ${ }^{6}$ In den früheren Notizen, die sich in ihrer großen Mehrzahl auf den Venedig-Aufenthalt beziehen, sucht man die Bezeichnung "Roman" dagegen vergeblich. Stattdessen finden sich "Reisetagebuch" (S. 7) und "Tagebuch« (S. 8), "große Erzählung«, »längere Erzählung« (S. 363), Novelle (vgl. S. 364) und »Prosa« (S. 364). Im Rahmen dieser übergeordneten Bezeichnungen wird das Material teils erzählerisch, teils dramatisch schematisiert. So ist zum einen von "Geschichte" (S. 125) und Anekdote (vgl. S. 121) die Rede, zum anderen vielfach von "Scene« (S. 22 und passim); im Übrigen gibt es eine Vorliebe für Begriffe, die zwischen literarischer Form und Lebensform changieren und sich sowohl erzählerisch als auch dramatisch akzentuieren lassen: vor allem "Abenteuer" (S. 9 und passim) und »Erlebniss" (S. 11 passim), aber auch "Situation" (S. 32) und "Episode« (S. 117 und passim).

\footnotetext{
5 Vgl. Hofmannsthals Brief an seinen Vater vom 1. Oktober 1912, in dem es heißt: »Ich arbeite fleißig. Wie lang man eigentlich braucht, um einen Roman zu schreiben." (SW XXX Roman, S. 364)

6 Vgl. SW XXX Roman, S. 127. Zitate aus dem »Andreas«-Konvolut werden künftig nach dieser Ausgabe im fortlaufenden Text mit Seitenzahlen belegt.
}

168 Inka Mülder-Bach 
Eine solche Offenheit ist bei Hofmannsthal an sich nicht ungewöhnlich. Denn Stoffe und Motive sind in seinem Werk häufig allotropisch; ${ }^{7}$ wie bestimmte chemische Elemente können sie in zwei oder mehr Formen vorkommen, die sich in ihrer Struktur unterscheiden. Morton Princes Studie »The Dissociation of a Personality « (1906), die eine wesentliche Anregung des "Andreas" war, hat Hofmannsthal dazu inspiriert, die Figur der gespaltenen Frau noch vor den ersten Notizen zum "Andreas" dem Dramenentwurf »Dominik Heintls letzte Nacht« (1906/07; 1913) zu integrieren, der seinerseits durch Balzacs Roman "Eugénie Grandet« angeregt wurde. ${ }^{8}$ Und so wie ein Stoff oder Thema in verschiedenen Genres Gestalt annehmen kann, können Texte verschiedener Genres auseinander hervorgehen bzw. ineinander transformiert werden - aus der Operndichtung »Die Frau ohne Schatten« (1919) geht das gleichnamige Märchen (1919) hervor, die Novelle »Lucidor" (1910) wird zu einem Ausgangspunkt des Librettos der »lyrischen Komödie« »Arabella« (1927) usw. Doch das "Andreas«-Konvolut bietet ein etwas anderes Bild. Die verschiedenen Gattungsbezeichnungen und Gestaltungsoptionen, die in den Aufzeichnungen erwogen werden, lösen einander nur teilweise ab; zum größeren Teil stehen sie gleichberechtigt nebeneinander. Auch der fragmentarische Hauptentwurf, in dem die formalen Möglichkeiten, die in den Notizen in der Regel nur genannt, nicht aber durchgespielt werden, zur Realisierung anstehen, trifft hier keine Entscheidung. Vielmehr ist er dadurch charakterisiert, dass sich verschiedene formensprachliche Impulse und Tendenzen verschränken und durchkreuzen.

Wie der Fragmentcharakter des "Andreas« ist die Heterogenität dieser Impulse in die Tradition der »Romanentwürfe[] der Frühromantiker « ${ }^{9}$ und der frühromantischen Theorie des Romans als universalpoetisches Medium der Vereinigung der Gattungen gestellt worden. Doch wenn Hofmannsthal im "Andreas" - unter anderem - an den frühromantischen Roman anzuschließen sucht, so ist das Fragmentarische des TexS. 485 .

7 Vgl. Hugo von Hofmannsthal, Über Charaktere im Roman und Drama. In: GW E, 485

8 Vgl. SW XVIII Dramen 16, S. 321 und Erläuterungen, S. 538f.; vgl. ferner Mayer, Die Grenzen des Textes (wie Anm. 3), S. 78.

9 Günter Schnitzler, Quellendichte und Unabschließbarkeit: Zu Hofmannsthals "Andreas«-Roman. In: Ralf Bogner u.a. (Hg.): Realität als Herausforderung. Literatur in ihren konkreten historischen Kontexten. Festschrift für Wilhelm Kühlmann zum 65. Geburtstag. Berlin/New York 2011, S. 447-462, hier S. 459. 
tes Ausdruck von spezifischen Problemen, die nicht schon dadurch zum Ausweis romantischer Traditionslinien werden, dass sie ungelöst blieben. Diese Probleme lassen sich am Text konkretisieren und betreffen das Verhältnis zwischen den beiden Handlungsräumen Kärnten und Venedig ebenso wie die erzählerische Gestaltung dieser Handlungsräume selbst. Hermann Broch hat Hofmannsthals Erzählprosa als "szenische Epik $«^{10}$ bezeichnet und den Fragmentcharakter des "Andreas" darauf zurückgeführt, dass Hofmannsthals "Bildmaterial« auf das "Milieuhafte" beschränkt bleibe, auf Elemente des "Landschaftlichen" und "Szenenhaften«, aus dem sich »der Aufbau eines Romanwerkes" allein »nicht bestreiten « lasse. ${ }^{11}$ Man muss sich der Wertung nicht anschließen, um anzuerkennen, dass Broch einen wichtigen Punkt trifft. Denn in der Tat hat Hofmannsthals Erzählprosa, wo sie nicht Landschaftliches beschreibt, eine starke Tendenz zum Szenisch-Dramatischen. Das manifestiert sich im »Andreas" zum einen in einer Erzählweise, die von Mischformen geprägt ist. Zum anderen zeichnet sich im Textaufbau ein Konflikt zwischen Episch-Romanhaftem und Dramatisch-Komödiantischem ab, der mit dem Zusammenhang zwischen literarischen Gattungen und kulturellen Topografien zu tun hat. Was Broch als Beschränkung auf "Milieuhafte[s]" - also auf Genrehaftes - beschreibt, hat, anders als Broch nahelegt, vielleicht weniger mit der Begabung des Autors als mit diesem Zusammenhang zu tun.

Von dem Hauptentwurf aus zurückblickend, deutet sich die entscheidende Bruchlinie des Textaufbaus schon in dem frühesten Entwurf an, in dem unter der Überschrift "Das Reisetagebuch des Herrn von N." eine zwischen Ich- und Er-Form wechselnde Erzählerinstanz von einer »Bildungsreise« nach Venedig berichtet.

Ich erinnere mich an die Dinge ganz genau. Hatte immer sehr gutes Gedächtnis. Bekam bei den Schulbrüdern das große Fleißkreuz weil ich die oester[reichischen] Regenten vor u. rückwärts aufsagen konnte. Auch alle Dienstmädchen meiner Mutter habe mir gemerkt und alle Mineralien meines Großvaters und die Namen des Sternbilds Orion.

10 Hermann Broch, Hofmannsthal und seine Zeit. In: Ders., Kommentierte Werkausgabe. Hg. von Paul Michael Lützeler. Bd. 9/1: Schriften zur Literatur 1: Kritik. Frankfurt a.M. 1975, S. 111-284, hier S. 249.

11 Hermann Broch, Hugo von Hofmannsthals Prosaschriften. Zweite Fassung. In: Ebd., S. 289-300, hier S. 292.

170 Inka Mülder-Bach 
Gründe der Bildungsreise. Maler. Große Namen. Paläste. Sitten im Salon. entréegespräche. Scheinen. Gefallen. Vorher von Venedig gewußt: Onkel hatte Bekannten dessen Verwandter in Oubliettes gestürzt (mit Nägeln u. Rasiermessern) ferner:

Ankunft: Morgengrauen: hungrig kühl, will sich um Unterkunft umschaun. Schauspielergesellschaft am Strand wartend. Eine coquettiert mit ihm, vom Schoß ihres Collegen herab. Gehe durch ein paar Gassen. Der halbnackte Herr. (S. 7)

Die auch optisch zweigeteilte Notiz hat zwei Pole: Herkunft einerseits und Ankunft in Venedig andererseits. Um den Pol der Herkunft lagern sich die Motive stupendes Gedächtnis und beliebiges Wissen, Familie, äußerliche Bildung und Reise; um den Pol der Ankunft die "Schauspielergesellschaft« und das Theater.

Diese Motive lassen sich unschwer den beiden Handlungsräumen Kärnten und Venedig zuordnen, zwischen denen der Hauptentwurf aufgespannt ist. In der frühesten Kapiteleinteilung (vgl. S. 12) entsprach deren Reihenfolge der fiktiven Chronologie der Reise, die "Finazzerhof«Episode sollte also dem Ankunftskapitel in Venedig vorausgehen. Es folgten andere Dispositionen, bis Hofmannsthal sich entschloß, das phänomenale Gedächtnis, das der Protagonist des Hauptentwurfs mit seinem Vorgänger Herrn von N. teilt, nicht nur zum Motiv, sondern zu einem Movens und Medium des Erzählens zu machen. So wird der Kärntenteil nunmehr als Vorgeschichte in Form einer langen Rückblende vermittelt, die nach dem Ankunftskapitel in Venedig eingeschoben ist und durch eine Intrusion ausgelöst wird, ein zwanghaftes Wiedererinnern, das den Protagonisten überfällt. Diese Anordnung eröffnet zugleich die Möglichkeit, mit der Ankunft in Venedig zu beginnen und am Anfang medias in res zu gehen.

"Das geht gut«, dachte der junge Herr Andres von Ferschengelder, als der Barkenführer ihm am $7^{\text {ten }}$ September 1778 seinen Koffer auf die Steintreppe gestellt hatte und wieder abstieß, »das wird gut, läßt mich da stehen mir nichts dir nichts, einen Wagen gibts nicht in Venedig, das weiß ich, ein Träger, wie käme da einer her, es ist ein öder Winkel, wo sich die Füchse einander gute Nacht sagen. Als ließe man einen um $6 \mathrm{~h}$ früh auf der Rossauerlände oder unter den Weißgärbern aus der Fahrpost aussteigen, der sich in Wien nicht auskennt. Ich kann die Sprache, was ist das weiter, deswegen machen sie doch aus mir, was sie wollen! Wie redet man denn wildfremde Leute an, die in ihren Häusern schlafen - klopf ich an und sag: Herr Nachbar?« - er 
wußte er würde es nicht tun - indem waren Schritte hörbar, scharf und deutlich in der Morgenstille auf dem steinernen Erdboden es dauerte lange bis sie näher kamen, dann trat aus einem Gäßchen ein Maskierter hervor, wickelte sich fester in seinen Mantel nahm mit beiden Händen ihn zusammen und wollte quer über den Platz gehen. (S. 40)

»Das geht gut«, »das wird gut«: Bereits im ersten Gedankenzitat des Protagonisten steht auch der mögliche Gang und Ausgang der Geschichte selbst zur Diskussion. Ob diese für den Protagonisten gut (gehen) wird, bleibt offen, dass sie hier und jetzt gut geht, wird schon durch den sprachlichen Gang seiner Gedanken dementiert. Zwei Anläufe braucht der Ankömmling, um nicht zu sagen, worum es (redensartlich) geht: 'Das geht nicht gut‘, >das wird nicht gut gehen`, >der ist gut, läßt mich hier stehen`. Und so wie übliche Redewendungen und die Lokaldeixis im doppelten Anlauf des ersten Gedankengangs verfehlt werden, werden sie im weiteren Fortgang entstellt. Denn in öden Winkeln pflegen entweder Wölfe - wie es im ersten Entwurf im Übrigen noch hieß (vgl. S. 327) - oder Füchse und Hasen sich oder einander gute Nacht zu sagen, aber dass "sich die Füchse einander gute Nacht sagen«, dürfte nicht einmal in den verlassensten Gegenden vorkommen. Während der Protagonist sich also versichert, die fremde Sprache zu können, wird gleichzeitig vorgeführt, wie er sich bei der Ankunft in der Fremde im eigenen Idiom verirrt.

Indem der Text seinen Einsatz mit einer Ankunft macht, rekurriert er auf eine paradigmatische Anfangsfigur, deren reflexives Potenzial auf der Hand liegt. ${ }^{12}$ Auch in der Literaturgeschichte von Venedigreisen kommt der Ankunft eine besondere Bedeutung zu. Doch anders als es zumindest so lange üblich war, wie Venedig allein mit dem Schiff erreicht werden konnte, verzichtet Hofmannsthal im "Andreas" - wie schon in dem Prosatext "Erinnerung schöner Tage» (1908) - auf eine Beschreibung der Annäherung an und Einfahrt in die Stadt. ${ }^{13}$ Unvermittelt und noch ehe er es sich versehen hat, ist der Protagonist frühmorgens vom Barkenführer irgendwo auf einer Treppe abgesetzt worden. Daraus ergibt sich eine Lücke, die Hofmannsthal niemals geschlossen hat. Denn wie

12 Vgl. Aage Hansen-Löve/Annegret Heitmann/Inka Mülder-Bach (Hg.), Ankünfte. An der Epochenschwelle um 1900. München 2009.

13 Vgl. Bernhard Dieterle, Die versunkene Stadt. Sechs Kapitel zum literarischen VenedigMythos. Frankfurt a.M. u.a. 1995, S. 387f. und S. $399 f$. 
der Protagonist von Kärnten nach Venedig gelangt, wird nicht erzählt. Was damit ausgespart bleibt, ist nicht nur eine geografische Route, sondern der Übergang von einem epischen kodierten Erzählraum zu einem dramatisch kodierten Schauplatz.

Die unvermittelte Ankunft in Venedig korrespondiert mit dem Anfang medias in res, den Hofmannsthal szenisch-theatralisch ausgestaltet. Szenisch ist schon das anfängliche Selbstgespräch des Protagonisten, das vom Gedankenzitat in den inneren Monolog übergeht. Mit dem akustisch sich ankündigenden Auftritt der Maske, die aus der Kulisse einer kleinen Gasse hervortritt, um den Platz zu überqueren, wird der "steinerne[] Erdboden« (S. 40) zum Bühnenboden und der Platz auch im buchstäblichen Sinn zum Schauplatz. Denn als der Mantel, in den die Maske gewickelt ist, wie ein Vorhang "vorne« aufgeht, steht vor Andreas ein Herr, der »im bloßen Hemde war, darunter nur herabhängende Kniestrümpfe die die halben Waden bloß ließen und Schuhe ohne Schnallen.« $(\text { S. } 41)^{14}$

Angesichts der Theaterleidenschaft des Protagonisten, von der erstmals beim Übergang in die Rückblende die Rede ist, liegt es nahe, diese »Bühne als Traumbild« zu lesen - und zwar nicht nur mit Hofmannsthal, ${ }^{15}$ sondern ebenso mit Freud. Denn Freud zufolge "dramatisierk ${ }^{16}$ ja der Traum selbst: "[E]in Gedanke, in der Regel der gewünschte, wird im Traume objektiviert« und »als Szene dargestellt« «, die der Träumende zu erleben glaubt; und eben dieses an die szenische Darstellung geknüpfte halluzinatorische Erlebnis ermöglicht die Wunscherfüllung. Darüber hinaus spielt Andreas in den wie traumhaft projizierten Szenen mit. So wie der Träumende Freud zufolge in aller Regel in seinem Traum vorkommt, sieht Hofmannsthals Protagonist nicht nur einen Schauplatz, sondern agiert mit auf der Bühne und wird dabei vom Erzähler beobachtet:

14 Dass der Schauplatz Venedig wie eine Kulisse wirkt und die Venedig-Episoden wie Bühnenbilder gebaut sind, ist ein Topos der Forschung. Vgl. u.a. Corbineau-Hoffmann, Der Aufbruch ins Offene (wie Anm. 2), S. 179-183; Dieterle, Die versunkene Stadt (wie Anm. 13), S. $412 \mathrm{ff}$.

15 Die Nähe der Venedig-Szenen zu Hofmannsthals Essay »Die Bühne als Traumbild« ist verschiedentlich herausgestellt worden. Vgl. u.a. David H. Miles, Hofmannsthal's Novel "Andreas". Memory and Self. Princeton 1972, S. 178f.; Sabine Straub, Zusammengehaltener Zerfall. Hugo von Hofmannsthals Poetik der Multiplen Persönlichkeit. Würzburg 2015, S. 340-349.

16 Sigmund Freud, Die Traumdeutung. In: Ders., Studienausgabe. Hg. von Alexander Mitscherlich, Angela Richards und James Strachey. Bd. II. Frankfurt a.M. ${ }^{3} 1972$, S. 74.

17 Ebd., S. 511. 
Andres tat einen Schritt vor und grüßte, die Maske lüftete den Hut und zugleich die Halblarve die innen am Hut befestigt war. Es war ein Mann der vertrauenswürdig aussah und nach seinen Bewegungen und Manieren gehörte er zu den besten Ständen. Andres wollte sich beeilen, es dünkte ihn unartig, einen Herrn, der nachhaus ging zu dieser Stunde lang aufzuhalten, er sagte schnell daß er ein Fremder sei, eben angekommen [...]. (S. 40)

Einerseits handelt es sich um eine interne Fokalisierung des Geschehens; entsprechend wird die Maske aus der Sicht des Protagonisten beschrieben. Andererseits steht dieser mit auf dem Schauplatz und ist als Schauspieler in das Geschehen involviert. Ins Präsens versetzt, könnte man sich den ersten Satz des Zitats ebenso gut als Regieanweisung vorstellen. "Im Anfang liegt das Ganze prädestiniert [...]. Der Anfang entschleiert die Gebärde des Schreibenden«, lautet eine Aufzeichnung Hofmannsthals aus dem Jahr 1909. ${ }^{18}$ Obwohl am Anfang des "Andreas" Gang und Ausgang des Ganzen angesprochen werden, hat die Ankunftsszene offenkundig nicht die prädestinierende Kraft, die Hofmannsthal einem Anfang zuschreiben wollte. Die dramatisch-erzählerische Doppelphysiognomie aber, die das Arrangement der Szene kennzeichnet, bleibt auch im Fortgang des Ankunftskapitels von konstitutiver Bedeutung und manifestiert sich auf verschiedenen Ebenen. Dazu gehört, dass die Handlung in Form von kleinen Szenen voranschreitet, in denen eine begrenzte Anzahl von Figuren wie dramatis personae vor den Augen des Protagonisten und zugleich mit ihm als Mitspieler auf- und abtreten. Dazu gehört die Koordination der Szenenfolge mit dem Wechsel von Schauplätzen, die Ausstaffierung mit Kostümen und Requisiten und das stumme Spiel der Physiognomie und Gesten. Und dazu gehört der Name des Viertels San Samuele, in dem Andreas an- und unterkommt. So heißt die Pfarrgemeinde, in der Giacomo Casanova geboren wurde, und so heißt das Theater, an dem Casanova und seine Eltern tätig waren, an dem Carlo Goldoni, der das Theater einige Jahre leitete, viele seiner Komödien zur Aufführung brachte und das später auch zur Bühne der Erfolge von Goldonis Rivalen Carlo Gozzi wurde. Im Namen San Samuele verdichten sich Intertexte der Erzählung, Inspirationsquellen ihres Autors sowie auf erotische Abenteuer und das Theater gerichtete Wunschenergien des Protagonisten, der dann ja auch prompt direkt gegenüber dem The-

18 GW RA III, S. 499.

174 Inka Mülder-Bach 
ater in dem Haus einer verarmten adligen Familie unterkommt, deren Mitglieder allesamt im Theater beschäftigt sind.

Das »Kärnten«-Segment des Hauptentwurfs führt nicht nur in einen anderen geografischen und landschaftlichen Raum, den Hofmannsthal der südlich gelegenen Lagunenstadt als nördliches Gebirge auch in seiner Komödie "Cristinas Heimreise» (1910) entgegensetzt. Mit dem Finazzerhof wird ein anderer Erzählraum aufgemacht. Er öffnet sich als ein Gedächtnisraum, in den der Protagonist wider Willen versetzt wird, als er sich am Fenster seines Zimmers in Venedig und beim Blick auf das Theater einer Assoziationskette überlässt, die ihn von dem Entwurf eines Briefs an die Eltern über das Volkstheater seiner Kindheit zu den »drei unheilvollen Tagen in Kärnten« führt:

[...] ob er wollte oder nicht mußte er sich an alles erinnern haarklein und von Anfang an; so kam es über ihn jeden Tag einmal, früh oder abends. Er hätte sein Gedächtnis verfluchen mögen. Wie in der Klosterschule mit den Kaisern und den Sternbildern im Gürtel des Orion (S. 46).

Mit den Namen der Kaiser und der Sterne im Oriongürtel knüpft diese Textstelle an "Das Reisetagebuch des Herrn von N." an und macht die Pathologie explizit, die in dem "sehr gute[n] Gedächtnis« (S. 7) der Hauptfigur dieses Entwurfs bereits angelegt ist: Andreas leidet größtenteils an Reminiszenzen. Im Text heißt es, dass die erinnerte Zeit durch "Andres hindurch" geht, dass er gezwungen ist, sie "wieder durchzumachen" (S. 49); was er dabei wieder durchmachen muss, ist unter anderem ein Traum, in dem er in ein "Durchhaus" gerät, in dem er »durch alle schiefen und queren Situationen seines Kindes- und Knabenlebens [...] wieder hindurch" musste (S. 64). ${ }^{19}$ Das Durchmachen und Durchgehen hat den Charakter eines Wiederholens, in dem das Durcharbeiten nicht gelingen will, der Wiederholungszwang nicht gebrochen wird. Die Erinnerung schafft also keine epische Distanz. Doch versetzt sie in einen Gedächtnisraum, in dem traditionelle epische und romanhaft-romantische Motive wiederkehren und mit der Erprobung narrativer Formen der Subjektivierung und szenischer Darstellungsverfahren sowie mit einer Fallgeschichte gekreuzt werden, die man auch als Studie über die

19 Vgl. zur Topografie dieses Traums Dieterle, Die versunkene Stadt (wie Anm. 13), S. 520 . 
Perversionen eines dekadenten Katholizismus samt seiner Bildquellen lesen kann. ${ }^{20}$

Zu den episch-romanhaften Motiven gehören Reise, Ritt und der Chronotopos des Weges; ferner der Themenkomplex von Herkunft, Genealogie, Verwandtschaft und Familie; und natürlich der »Ritter« (S. 56) samt Castell, Rittergeschlecht, Wappen und Schwert. Ein solches, nämlich das "zweischneidig Schwert von zartester Wollust und unseglicher Sehnsucht" (S. 46), das Andreas einst im Volkstheater beim Anblick des blauen Schuhs der Prinzessin durchfuhr, fungiert als Signalwort, das ihn aus der Erinnerung an seine Kindheit in die Herberge "zum 'Schwert in Villach" und in die Begegnung mit dem Bedienten Gotthelf versetzt.

Er war wieder in der Herberge zum "Schwert« in Villach, nach einem scharfen Reisetag, und wollte zu Bett gehen, da, schon auf der Treppe präsentierte sich ihm ein Mensch als Bedienter oder Leibjäger. Er: er brauche keinen, reise allein [...]. Der andere darauf läßt ihn nicht los, steigt Stufe für Stufe mit, [...] tritt dann in der Tür quer auf die Schwelle, daß Andres sie nicht zu machen kann: daß es nicht schicklich wäre für einen jungen Herrn von Adel, ohne Bedienten zu reisen [...]. (S. 47)

Der Unmittelbarkeit der Wiedererinnerung entsprechend gibt es erneut einen Anfang medias in res und den Aufbau einer Szene, in diesem Fall mit dem Schauplatz Gasthaus und dem Bühnenbild Treppe, wie man das seit "Silvia im Stern« (1909) aus Hofmannsthals Komödien kennt. Zugleich gibt es den Ansatz zur Notation einer dramatischen Sprecherrolle, deren direkte Rede aber vom Erzähler zugunsten der indirekten zurückgedrängt wird, sodass der Text selbst gewissermaßen auf der "Schwelle« zwischen mimesis und diegesis zögert. Die Vorliebe für diese eher schwerfällige Form der indirekten Wiedergabe von Figurenrede ist schon im Ankunftskapitel bemerkenswert. Im weiteren Fortgang des KärntenAbschnitts nimmt sie einen ganz ungewöhnlich breiten Raum ein. Insbesondere die Reden, in denen die unschuldige Jungfrau Romana und der teuflische Diener Gotthelf von ihrem Leben erzählen, werden seitenlang mit inquit-Formeln und im Konjunktiv mitgeteilt. Die indirekte Rede ist

20 Vgl. zu den Bildquellen und zur Ikonografie der Kärnten-Segmente Waltraud Wiethölter, Hofmannsthal oder Die Geometrie des Subjekts. Psychostrukturelle und ikonographische Studien zum Prosawerk. Tübingen 1990, S. 131-167; W.G. Sebald, Venezianisches Kryptogramm. Hofmannsthals »Andreas«. In: Ders., Die Beschreibung des Unglücks. Zur österreichischen Literatur von Stifter bis Handke. Frankfurt a.M. 1994, S. 61-77. 
hier also eine transponierte Narration, wobei der Wortschwall, in dem Gotthelf von seiner Jugendzeit als Leibjäger berichtet, und der Assoziationsstrom, der Romana vom Adler des Großvaters zu dem inzestuösen Verhältnis der Eltern führt, von dem Erzähler nicht dem eigenen Duktus angeglichen werden.

Den [Adler] hab der Großvater heim gebracht noch fast nackt denn Adlerhorst ausnehmen das wäre dem Großvater sein Sach gewesen, sonst habe er bereits nichts getrieben, aber oft tageweit reiten dann hinaufsteigen einen Horst aufspüren [...] hinauf und ein Nest ausnehmen oder an einem Strick sich herablassen kirchturmtief dies sei sein Sach gewesen und schöne Fraun heirathen, das habe er viermal gethan und nach jeder Tod allemal eine noch schönere und allemal aus der Blutsfreundschaft denn habe er gesagt über's Finazzerische Blut gehe ihm nichts. Wie er den Adler da gefangen sei er schon 54 gewesen und an 4 Kirchenleitern 9 Stunden über dem schrecklichsten Abgrund gehangen darauf aber seine letzte Frau gefreit. (S. 57)

Auch in einer Ausgabe, die die fehlenden Satzzeichen ergänzt, hätten wir es hier mit einem transponierten Erzählen ohne Punkt und Komma zu tun. Das entbehrt nicht der Komik, doch um einen parodistischen »Transfer«, in dem »das Erzählen im traditionellen Sinn anderen, nämlich Figuren des Romans, überlassen ${ }^{21}$ wird, handelt es sich schon deshalb nicht, weil diese Form der transponierten Narration, die einem stream of consciousness in indirekter Rede gleicht, kein traditionelles Erzählen ist. Eher schon wird jene assoziative Verkettung von »beweglichen Worten« (S. 63) und »schönen Wendungen" (ebd.) gespiegelt, von denen im Zusammenhang mit dem Brief die Rede ist, den Andreas "in seiner Einbildung" (ebd.) an die Eltern schreibt und in dem er ihnen mitteilt, dass er seine Reise abzubrechen und Romana zu heiraten gedenkt. "Die Gedanken strömten ihm« (ebd.), heißt es über die Sprache dieses eingebildeten Briefs. Wie diese "schöne Kette« (ebd.) lautet, erfährt der Leser allerdings nicht. Denn auch hier verzichtet Hofmannsthal auf den inneren Monolog, um die Gedanken erneut nur in indirekter Rede mitzuteilen. An dieser Stelle gibt die indirekte Rede den Duktus der direkten jedoch nicht nur nicht wieder, vielmehr fällt der Erzähler sich gleichsam selber ins Wort und weist ausdrücklich darauf hin, dass er diesen

21 Corbineau-Hofmann, Der Aufbruch ins Offene (wie Anm. 2), S. 175.

Genremischung und Gattungskonflikt 177 
Duktus verfehlt: "Der Brief den er [Andreas] in Gedanken schrieb, war weit über dieser dürftigen Inhaltsangabe.« (Ebd.)

So wenig Andreas den eingebildeten Brief wirklich schreibt, so wenig teilt er sich erzählerisch mit. Was wir von seiner Lebensgeschichte erfahren, wird vermittelt über die Darstellung jener alterierten Zustände berichtet, in denen der Protagonist von seiner Biografie eingeholt wird. Hier, in Träumen, Halluzinationen, Wiedererinnerungen und Zuständen der Spaltung, kehren die Traumata und Schrecken der Kindheit wieder: Die "Spiegelgasse« mit der Wohnung der Eltern, »der Blick seines ersten Katecheten« und dessen "gefürchtete kleine feiste Hand«, die anzüglichen Reden des Knaben mit dem »widerwärtige[n] Gesicht» (S. 64), die Marter und das Martyrium von Katze und Hund. Das ergibt zusammengenommen den pathologischen »Fall« eines durch sexuellen Missbrauch traumatisierten jungen Mannes, der zwischen Größenwahn und Minderwertigkeitskomplexen, Autoritätshörigkeit und Aggressivität hin und her gerissen und hinter der mühsam aufrechterhaltenen Fassade kleinadliger Normalität von sadomasochistischen Impulsen beherrscht und von Dissoziation bedroht ist. Aus psychoanalytischer Sicht passt dazu, dass die Erinnerungsfragmente sich nicht zu einer erzählbaren Geschichte fügen. Denn das Nicht-Erzählen-Können ist Freud zufolge geradezu »das notwendige, theoretisch geforderte Korrelat火 ${ }^{22}$ der Neurose.

Das Genre der Fallgeschichte wird auch durch die Intertexte aufgerufen, die in der Darstellung der alterierten Gefühlszustände des Protagonisten ins Spiel kommen. An sie lehnt sich Hofmannsthal nicht nur motivisch, sondern auch erzähltechnisch an. So macht er in verschiedenen Varianten häufig von der vergleichenden Wendung sihm war (als)< Gebrauch, die im Zuge der Psychologisierung der Literatur im 18. Jahrhundert Eingang in das Erzählen fand. In Karl Philipp Moritz' Roman "Anton Reiser« oder später in Georg Büchners Erzählung "Lenz« avancierte diese Wendung zu einem zentralen Mittel der Darstellung psychischen Erlebens und hatte im frühen 20. Jahrhundert nicht nur bei Hofmannsthal, sondern auch bei Musil noch einmal Konjunktur, bevor

22 Sigmund Freud, Bruchstück einer Hysterie-Analyse. In: Ders., Studienausgabe (wie Anm. 16), Bd. VI: Hysterie und Angst ( $\left.{ }^{8} 1994\right)$, S. 96.

178 Inka Mülder-Bach 
sie endgültig durch flexiblere Formen - wie die erlebte Rede und den inneren Monolog - verdrängt wurde. ${ }^{23}$

Wer füttert jetzt in der Nacht einen Hund dachte es in ihm und zugleich war ihm gepreßt zumut als müsse er nochmals zurück in seine Knabenzeit als er noch das kleine Zimmer neben den Eltern hatte und sie durch den in die Wand eingelassenen Kleiderschrank mußte abends reden hören er mochte wollen oder nicht. (S. 61)

Andres war aus dem Bett und zog sich an, aber dabei war ihm zumut wie einem Verurteilten, den das Pochen des Henkers geweckt hat; der Traum hing noch zu sehr um ihn, die gestrige Nacht - ihm war als habe er etwas Schweres begangen und nun komme alles ans Licht. (S. 65)

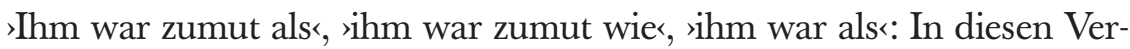
gleichskonstruktionen erscheint das Subjekt des Erlebens grammatisch in der Position des Dativobjekts, und zwar des Objekts des eigenen Gefühls, des eigenen psychischen Erlebens, auf das der Vergleich zielt. ${ }^{24}$ Ähnlich wie in der erlebten Rede bleibt in der Schwebe, wer spricht zwischen "äußerer Beschreibung und »innerer [...] Ausdeutung « ${ }^{25}$ ist nicht zu unterscheiden. Und erneut handelt es sich um eine Erzählform, die zum Szenisch-Theatralischen tendiert. Im ersten Beispiel erinnert der Vergleichssatz an eine vergangene Situation, die, als sie erlebt wurde, von dem Gefühl begleitet war, um dessen Darstellung es geht; im zweiten Zitat wird eine Situation fingiert - die Situation des Verurteilten, den das Pochen des Henkers geweckt hat -, die, wenn sie wirklich wäre, mit dem Gefühlszustand verknüpft wäre, der dargestellt werden soll. In beiden Fällen schlägt die Vergleichskonstruktion »eine imaginäre Bühne auf, die es ermöglicht, das Gefühl narrativ zu theatralisieren. $\aleph^{26}$

So wie die "Kärnten«-Rückblende mit einer »Treppen«-Szene beginnt, in der Andreas und sein Diener Gotthelf ganz buchstäblich nach oben steigen, mündet sie in das Bild einer erhabenen Gebirgslandschaft mit einem in der Höhe kreisenden Adler und einem seelischen Aufschwung, in dem Andreas ein mystisches Erlebnis der Einheit hat. Damit wird den

23 Vgl. Johannes F. Lehmann, »Es war ihm, als ob...« Zur Theorie und Geschichte des »erlebten Vergleichs«. In: ZfdPh 132, 2013, S. 481-498.

24 Vgl. ebd., S. 483-488.

25 Helmut Müller-Sievers, Desorientierung. Anatomie und Dichtung bei Georg Büchner. Göttingen 2003, S. 156.

26 Lehmann, »Es war ihm, als ob...« (wie Anm. 23), S. 488. 
Handlungsräumen Kärnten und Venedig auch in ästhetischer Hinsicht eine Höhendifferenz eingeschrieben. Zugleich wird die Erzählung auf das Telos einer Rückkehr zu und Vereinigung mit Romana ausgerichtet, an dem Hofmannsthal bis in späteste Notizen hinein festgehalten oder an das er (sich) noch in spätesten Notizen erinnert hat (vgl. S. 217).

Im Rahmen des übergreifenden Handlungsbogens, der damit konzipiert wird, fungiert Venedig nicht als das Ziel, als das es im diegetischen Universum in der von den Eltern entworfenen Bildungsreise konzipiert ist. Es bezeichnet eine Zwischenstation mit initiatorisch-therapeutischer Funktion. Während die Erinnerung an die Tage in Kärnten und die in ihnen aufgestiegenen Schrecken der Kindheit, in die sich der Protagonist seit seiner Abreise vom Finazzerhof jeden Tag versetzt sieht, den Wiederholungszwang nicht zu brechen vermag, soll im Labyrinth Venedigs eine Passage gefunden werden, die aus der Wiederholung hinausführt. Denn die Rückkehr in den Erzählraum Romanas und die Vereinigung mit derselben setzen offenkundig voraus, dass das explosive Gemisch der Fallgeschichte entschärft wird. Dass es dazu nicht kommt, dass Text und Held in Venedig stecken bleiben, ist bekannt. Die Frage ist, warum das ist und wie man das Verhältnis der Textsegmente beschreiben kann, wenn sie nicht durch den angedeuteten, aber nicht ausgeführten Handlungsbogen miteinander vermittelt werden.

Die im »Kärnten«-Abschnitt entfaltete Problematik der Zerstückelung, Entzweiung und Spaltung kehrt in Venedig in einer Handlung wieder, die unter anderem durch Morton Princes Studie über eine dissoziative Persönlichkeitsstörung inspiriert wurde. Im Rahmen dieser Handlung begegnet Andreas in der Gestalt des Malteserritters Sacramozo sowie in der Doppelfigur von Maria/Mariquita Aspekten, Spiegelungen und Doppelgängern seiner selbst. In dem auf die »Kärnten«-Rückblende folgenden zweiten zusammenhängenden Venedig-Segment werden diese Spiegelfiguren noch eingeführt. Was mit den sich dabei abzeichnenden Konstellation im Weiteren hätte geschehen sollen, darüber kann man nur spekulieren. In manchen Aufzeichnungen ist von wechselseitigen Verwandlungen die Rede, in anderen von Vereinigungen. Doch ganz abgesehen davon, dass die textuelle Dynamik der Fragmentierung der Vereinigung entgegenläuft, ist nicht einmal klar, wer gegebenenfalls mit wem hätte eins werden sollen: »die Subiecte [...] mit sich selber« (S. 119, 
vgl. auch 152), Andreas mit Maria (vgl. S. 108), die Venedig-Figuren untereinander (vgl. S. 119) oder Andreas mit Romana (vgl. S. 217).

Die Forschung hat das Verhältnis zwischen dem »Kärnten«-Abschnitt und den Venedigsegmenten zumeist unter dem Aspekt der Identitätsproblematik und also als ein psychologisches behandelt und dabei vor allem auf Gemeinsamkeiten hingewiesen. Für die einen wechseln zwar »Kulissen und das Ensemble«, nicht aber »Text und die Regie«; ${ }^{27}$ für andere beruht die Dynamik in beiden Handlungsräumen auf einer Überblendung von zeitgenössischen Perversions-, Hysterie- und Dissoziationskonzepten; ${ }^{28}$ aus einer dritten Sicht sind die Segmente schlicht "untereinander austauschbar ${ }^{29}{ }^{29}$

Wenn man das Verhältnis der Abschnitte poetologisch betrachtet, ergibt sich ein anderes Bild. Wie bereits erwähnt, führt Hofmannsthal die Bezeichnung "Roman« erstmals nach Abbruch der Arbeit an dem Hauptentwurf in Notizen zum Finazzerhof ein. Dem entspricht die epische Signatur des Erzählraums Kärnten ebenso wie der Name "Romana", der offenkundig ein sprechender ist. Er enthält eine ganze Wortfamilie, zu der neben Roman, Rom und Römerin auch das Heilige Römische Reich gehört, das in Gestalt des Bilderbuchs "aus der Zeit von Kaiser Maximilian dem Ersten« (S. 56) in der "Finazzerhof«-Episode evoziert wird. Ob der Umstand, dass es die Republik Venedig war, die dem »letzten Ritter« Maximilian I. den Durchzug nach Rom verwehrte, sodass er den Titel "Erwählter Römischer Kaiser» mit Billigung des Papstes in Trient annahm, in Hofmannsthals Fragment eine Rolle spielt, kann dahingestellt bleiben. Das Identitätsproblem des Protagonisten jedenfalls wird im Erzählraum Venedig nicht nur vor einer anderen Kulisse verhandelt als in der Gebirgswelt Kärntens, es wird ihm auch ein Drehbuch zugrunde gelegt, das sich an einem anderen Genre orientiert als dem des Romans. Dem Schauplatz entsprechend, nähert sich dieses Drehbuch jener "comedy of errors" - "sometimes farcical and sometimes tragic" - an, als die Prince die Fallgeschichte seiner dissoziierten Patientin bezeichnete. ${ }^{30}$ Damit aber wird das gesamte Material tendenziell neu konfiguriert. Das

27 Wiethölter, Hofmannsthal oder die Geometrie des Subjekts (wie Anm. 20), S. 169.

28 Vgl. Maximilian Bergengruen, Mystik der Nerven: Hugo von Hofmannsthals literarische Epistemologie des 'Nicht-mehr-Ich . Freiburg i.Br. 2010.

29 Straub, Zusammengehaltener Zerfall (wie Anm. 15), S. 332.

30 Morton Prince, The Dissociation of a Personality. A Biographical Study in Abnormal Psychology. New York/London/Bombay 1906, S. 7. 
ästhetische Gefälle zwischen Pathos und Bathos und der Abstieg von oben nach unten wurden schon genannt. Die Höhendifferenz findet ihr Komplement in einem Größenunterschied, sodass der untere Schauplatz Venedig auch von einer betonten Enge und Kleinheit ist. Die Rekonfiguration des Materials aber manifestiert sich auch darin, dass die erzählerische Sukzession in ein dramatisches Nebeneinander und der epische Weg in eine komödiantische Situation überführt wird.

Ein offenkundiges Symptom dieses Wechsels ist die Preisgabe des Tagesschemas - "Castell Finazzer: der erste Tag« (S. 53) und »(Der dritte und vierte Tag in Kärnten) « (S. 68) -, das die analeptische Erzählung des Aufenthalts in Kärnten strukturiert. Aber man kann sich die Umorganisation des Materials auch an den Transformationen verdeutlichen, die die Konzepte von Familie und Verwandtschaft erfahren. ${ }^{31}$ Der Name "Romana" umfasst nicht nur eine ganze Wortfamilie, er taucht in Hofmannsthals Euvre erstmals in einem Prosatext von 1898 auf, der den Titel »Die Verwandten« trägt. Schon hier wird der Name mit dem "Ordnungsschema der Lebensalter in Zusammenhang gebracht « ${ }^{32}$ Entsprechend bezieht sich Verwandtschaft im Erzählraum Kärnten und im Rahmen des »Romana«-Romans auf Herkunft, Abstammung und biologische Familie und wird primär als lineare genealogische Folge akzentuiert. In dieser Bedeutung wird Verwandtschaft zum einen im Hinblick auf Andreas zum Thema, dem, wenn er an Verwandte denkt, die Eltern, Großvater Ferschengelder sowie Leopold einfallen, den Onkel mit seiner kinderlosen legitimen Frau und seiner illegitimen Geliebten samt den illegitimen Nachkommen, nach dem Andreas angeblich schlägt. Zum anderen werden genealogische Verhältnisse an Romana und dem Geschlecht der Finazzer verhandelt, dessen Reproduktion inzestuös in-

31 Während der Herausgeber der »Kritischen Ausgabe« von den Genealogien, von denen das Fragment erzählt, auf das Vorhaben einer kontinuierlich erzählten »Lebens- und Bildungsgeschichte« (S. 305), eines »Entwicklungs- und Familienroman[s] (S. 311) beziehungsweise eines "groß angelegten Generationenroman[s]« (S. 309) schließt, wird in neuerer Forschung betont, dass das $»$ Denken an die Verwandtschaft« immer auch ein $» D e n k e n$ in Verwandtschaften" sei (Straub, Zusammengehaltener Zerfall [wie Anm. 15], S. 326); hier steht die "genealogische Konzeption des 'Andreas « im Dienst einer »Dynamik der Verräumlichung und Simultaneität der verwandtschaftlichen Relationen, wie sie im Modell der zusammengesetzten Persönlichkeit gegeben ist." (Ebd. S. 328). Beide Positionen übersehen, dass es gerade der Konflikt zwischen diesen unterschiedlichen Konzeptionen ist, von dem das Fragment gekennzeichnet ist.

32 Konrad Heumann, Romana. Hofmannsthal und die Utopie des einfachen Lebens. In: WW 60, 2010, S. 31-47, hier S. 42.

182 Inka Mülder-Bach 
nerhalb des eigenen »Bluts" erfolgt, wobei von den neun Geschwistern Romanas nur drei überlebt haben. In beiden Familien also gibt es erhebliche Unterbrechungen und Verwerfungen in der Reproduktionskette, die den Rekurs auf ein genealogisches Erzählmodell erschweren, um nicht zu sagen unmöglich machen.

In Gestalt der Familie des Grafen Prampero oder Gasparo - der Name wechselt bekanntlich - kommt Andreas auch in Venedig in Kontakt mit einem biologischen Verband, dessen Reproduktion gefährdet ist. Die Gefährdung ist allerdings finanzieller Art, weshalb die Jungfräulichkeit der jüngsten Tochter Zustina in einer Lotterie verlost werden soll; und der Zusammenhalt des Verbandes wird nicht durch das eigene Blut, sondern sozial vermittelt. Die Familienmitglieder des Grafen sind ebenso wie alle anderen Bewohner seines Hauses in der Komödie beschäftigt. Das ergibt ein Ensemble, das an die "Schauspielergesellschaft" (S. 7) des frühesten Entwurfs erinnert und in seiner Funktion der »lustige[n] Gesellschaft « ${ }^{33}$ in den Komödien Hofmannsthals vergleichbar ist, auf die Juliane Vogel in ihrem Aufsatz »Komische Schwärme. Zur Poiesis des Sozialen bei Hugo von Hofmannsthal« aufmerksam macht. ${ }^{34}$ Das Ensemble fängt den gefährdeten Protagonisten auf und lässt ihn im Durchgang durch ein komödienhaftes Milieu in eine Konstellation geraten, in der genealogisch-biologische Konzepte keine Rolle spielen. Was in dieser Konstellation interessiert, sind nicht diachrone Stammbäume, Ahnentafeln und Blutsbande, sondern synchrone Anordnungen. Hier treten Figuren auf, deren multiple Identitäten insofern das epische Inzestmotiv variieren, als sie eine "Familie in sich" (S. 135) bilden, und die zugleich als Elemente einer Kombinatorik fungieren, in der sich ihre Verwandtschaft mit anderen multiplen Figuren - das "Verwandtschaftsfluidum" (S. 35), wie Hofmannsthal das auch nennt - in wechselnden Beziehungsmustern manifestiert. Das bringt Verdoppelungen, Verwirrungen und Verwechslungen mit sich, mit denen die Komödie seit jeher ihre Intrigen bestreitet. Entsprechend knüpft auch Hofmannsthal selbst später vor allem mit Entwürfen zu Komödien oder der Komödie nahestehenden Formen an das »Andreas«-Fragment an. ${ }^{35}$

33 SW V, Dramen 3, S. 104.

34 Vgl. den gleichnamigen Aufsatz von Juliane Vogel in diesem Band.

35 Vgl. Mayer, Die Grenzen des Textes (wie Anm. 3), S. 80. Beispiele wären etwa "Caféhaus oder der Doppelgänger«, »Timon der Redner«, »Emporkömmling« und »Das Hotel«. 
Dass der Protagonist des Erzählfragments ein gewisses Talent zum Komödiantischen hat, erhellt schon aus dem Umstand, dass es ihm nach dem Urteil seiner Eltern an einem "Auftreten« (S. 69) mangelt. Darüber hinaus wäre er auch insofern in einer Komödie gut aufgehoben, als er eine »Unsicherheit im Wiedererkennen" hat und zum »Verwechseln ähnlicher Menschen" sowie zum "plötzliche[n] Anders-sehen eines Gesichtes«(S. 37) neigt. Das wäre in einer Komödie kein Symptom einer Pathologie, sondern eine subjektive Disposition, die das Komödienhafte potenziert. Nicht in die Komödienwelt scheint dagegen die Figur des Maltesers Sacramozo zu passen, der eher dem Venedig der Verschwörungen und Geheimbünde entsprungen zu sein scheint und zugleich als "Ritter« (S. 84) eine Brücke zu Kärnten bildet. Eine Notiz hält fest: "Goldoni (= die Welt Zustinas das völlig unmetaphysische) ist ihm furchtbar, Molière bedeutet ihm nicht viel.« (S. 107) Damit mag zusammenhängen, dass Andreas Sacramozo erstmals in einer zweigeteilten Szene begegnet, in der auf der einen Seite "der reiche Grieche und sein bettelhafter Neffe« ein "gemeines Schauspiel« aufführen, auf der anderen der vornehme "Ritter« briefeschreibend an einem Caféhaustisch sitzt, wobei es für Andreas "außer der Begreiflichkeit« liegt, dass das eine sich in unmittelbarer Nachbarschaft des anderen "abspielen« kann (S. 83f.). Aber selbst wenn Sacramozo als binnenfiktionaler Kritiker und möglicherweise sogar als konzeptueller Gegenspieler der Komödie entworfen wäre, kann er sich dieser im Hauptentwurf nicht entziehen. Die Szene seines ersten Auftritts, die sich auf einem freien Platz vor einer kleinen Caféboutik abspielt, zitiert Goldonis Komödie »La Bottega del Caffé«; $; 6$ in die Beschreibung seiner Erscheinung hat Hofmannsthal Züge des Gazettenschreibers Lavache aus dem im Zusammenhang mit der Komödie "Christinas Heimreise« (1910) entstandenen szenischen Fragment »Florinda und die Unbekannte" (1909) kopiert, das im Übrigen an einem ähnlichen Schauplatz beginnt. Vor allem aber hat auch Sacramozo eine doppelte Natur, auch er wird mit einem inneren »Doppelgänger« (S. 141 und S. 144) versehen, der mit der Doppelfigur Maria/Mariquita kommuniziert. Die Ahnenreihe dieser Doppelfigur ist tendenziell unabsehbar. ${ }^{37}$ Gerade in ihrem Mariquitaaspekt und als Cocotte aber ist sie min-

36 Vgl. Dieterle, Die versunkene Stadt (wie Anm. 13), S. 412.

37 Vgl. Aurnhammer, Hofmannsthals "Andreas" (wie Anm. 4), S. 291; Schnitzler, Quellendichte und Unabschließbarkeit (wie Anm. 9), S. 452-459.

184 Inka Mülder-Bach 
destens ebenso sehr durch die Komödie und die Commedia dell'Arte inspiriert wie durch Morton Prince, zumal dieser selbst ja eine Art "comedy of errors" schreibt. Sie hat die Erfindungsgabe und die Unternehmungslust der Komödienfiguren - schon der »Luftzug" (S. 83), der ihre Nähe signalisiert, bringt Schwung in die Dinge. Sie ist bis in ihr Reden und Denken hinein "ganz Pantomime" (S. 18) und könnte "ein verkleideter Mann [...] oder eine öffentliche Person $\aleph^{38}$ sein. Sie spielt mit ihren »trucs« (S. 10) die Streiche und landet die Coups, die für Hofmannsthal unverzichtbar zur Komödie gehören. ${ }^{39}$

Indem er seinen Protagonisten nach dem Kärntenaufenthalt in das Milieu der Theaterstadt Venedig versetzt und seine Identitätsproblematik in ein Drehbuch übersetzt, das sich an der Komödie orientiert, knüpft Hofmannsthal offenkundig an die Tradition des Bildungsromans und insbesondere an Goethes "Wilhelm Meisters Lehrjahre« an. Auch Wilhelms Theaterliebe wird aus Kindheitserlebnissen hergeleitet; auch er ist empfänglich für zeittypische Pathologien; auch er hat ein Trauma zu verarbeiten und gerät in die Wunschwelt einer abenteuerlichen "Schauspielergesellschaft«. Bei Goethe ist die Entwicklung, die der Protagonist nimmt, nicht von der des Romans zu trennen. So wie die "Lehrjahre« den Theaterroman der "Theatralischen Sendung « hinter sich lassen, lässt der Held der "Lehrjahre" die Schauspielerwelt, das Rollenmodell Hamlet und die Vorliebe für szenische Vergegenwärtigungen in einem Prozess hinter sich, in dem der Roman selbst seine Poetik im Verhältnis zum Drama reflektiert und klärt.

Auch bei Hofmannsthal wäre grundsätzlich vorstellbar, dass der Roman aus einer Auseinandersetzung mit dem Drama - in diesem Fall nicht der Tragödie, sondern der Komödie - erwächst, die sowohl im diegetischen Universum als auch im Diskurs des Erzählers beziehungsweise im Medium der Erprobung und Reflexion von narrativen Verfahren stattfindet. Zwar ist die Ausgangssituation in einer nicht unwesentlichen Hinsicht eine andere. Denn während der Weg, der Wilhelm von

38 GW E, S. 256. Der Text wird hier ausnahmsweise nach dieser Ausgabe zitiert. Anstelle der Lesart "verkleideter Mann" findet sich in der "Kritischen Ausgabe die Transkription "verkleidete Nonne« (SW XXX Roman, S. 91). Zur Abwägung dieser Lesarten und zu den Argumenten, die für die Transkription in GW sprechen, vgl. Aurnhammer, Hofmannsthals "Andreas" (wie Anm. 4) S. 279.

39 Vgl. GW RA III, S. 512f., S. 533 und S. 542; vgl. auch Karin Wolgast, Die Commedia dell'arte im Werk Hugo von Hofmannsthals. Århus 1990. 
Mariane zu Natalie führt, insofern ein Modell der erotischen Sozialisation des bürgerlichen Mannes bietet, als er im Bett einer Geliebten aus der Theaterbohème beginnt und in den Seelenbund einer enthaltsamen Ehe mündet, geht der Roman um Romana im »Andreas«-Fragment der Komödie chronologisch voraus. Am Modell des »Wilhelm Meister" gemessen, hat Hofmannsthals Held gleichsam vorzeitig sein romanhaftes Ziel gefunden. Er glaubt sich mit Romana vereint, ohne schon jemals "ein Weib [...] ohne ihre Kleider gesehen geschweige angerührt« zu haben (S. 49f.); er glaubt, in ihr den "Mittelpunkt« seiner »Seele« (S. 76) gefunden zu haben, bevor seine erotische Initiation und Sozialisierung im Medium der Komödie begonnen hat. Wenn Text und Held daher im Durchgang durch den Theater- und Abenteuerraum Venedig zu ihrer romanhaften Form hätten finden sollen, so wären sie am Ende gewissermaßen zu ihrem Ausgangspunkt zurückgekehrt. Doch wenn eine solche Entwicklung im "Andreas" angelegt ist, dann führt sie zu keiner Klärung. Im Fluchtpunkt der Notizen und Aufzeichnungen, die nach dem Abbruch der Arbeit an dem Hauptentwurf entstehen, steht der Roman, der aus der venezianischen Verwechslungs- und Doppelgängerkomödie hervorgehen soll. Im Fluchtpunkt des Hauptentwurfs aber steht die Komödie, in deren Milieu und Grundriss Held wie Materialien nach dem Aufenthalt im romanhaft signierten Raum Kärnten unvermittelt versetzt werden. Als Überschrift könnte man sich den Untertitel der »Lucidor«Novelle vorstellen: "Figuren zu einer ungeschriebenen Komödie« ${ }^{40}$

40 GW E, S. 173.

186 Inka Mülder-Bach 